\title{
Attitude face à la fin de vie et à la mort
}

\section{Christian Kind}

Prof. Dr méd., président de la sous-commission "Attitude face à la fin de vie et à la mort»

Le traitement, la prise en charge et l'accompagnement de patientes et de patients confrontés à leur propre mort est une mission centrale de la médecine qui exige un profond respect de la dignité et de la volonté autodéterminée des patients et une haute responsabilité éthique. L’ASSM a rédigé des directives médico-éthiques qui traitent de l'attitude face à la fin de vie et à la mort, dont la version définitive a été approuvée par le Sénat de l'ASSM le 17 mai 2018.

L'écho rencontré par les directives «Attitude face à la fin de vie et à la mort» témoigne de l'intérêt pour ce sujet: 118 prises de position, dont la plupart très détaillées et nuancées, sont parvenues au Secrétariat général de l'ASSM. 92 d'entre elles provenaient de sociétés de discipline, de commissions d'éthique, d'associations, d'institutions et des pouvoirs publics et 26 de personnes individuelles.

Dans la majorité des prises de position, les changements par rapport aux directives antérieures «Prise en charge de patientes et de patients en fin de vie» ont été accueillis favorablement, notamment l'élargissement du champ d'application, l'importance accrue accordée au dialogue et une meilleure prise en compte des proches. De même, l'approche nuancée de la sédation palliative a été saluée à de nombreuses reprises.

Sans surprise, les dispositions relatives à l'assistance au suicide ont suscité de vives critiques. Mais 19 avis (22\%) seulement, émanant en majorité de personnes individuelles, ont rejeté la proposition de l'ASSM, les uns jugeant les directives trop libérales (13), et les autres (4) pas assez.

Sur la base des résultats de la consultation, les organes de l'ASSM ont décidé de conserver dans une large mesure le projet de texte initial rédigé par la sous-commission et de limiter les modifications à quelques points de détail. Pour répondre à diverses demandes, les directives comportent à présent une justification éthique explicite pour la réglementation de l'assistance au suicide.

En plus des modifications dans le chapitre sur l'assistance au suicide, diverses clarifications et améliora- tions ont été apportées par rapport au texte mis en consultation. Il s'agit principalement de la clarification de la praticabilité des directives, également concernant les enfants de tous âges et les personnes souffrant de handicap mental ou polyhandicapées ainsi que la procédure en situation d'urgence. Par ailleurs, une demande concernant l'aspect linguistique a été prise en compte: les dénominations successivement masculines et féminines dans le texte seront alternées d'un chapitre à l'autre.

\section{Structure et contenu des directives}

Le préambule résume le contexte et les objectifs des directives. Celles-ci s'entendent comme un soutien destiné aux professionnels de la santé pour une approche de la fin de vie et de la mort de leurs patientes et patients, orientée selon les principes des soins palliatifs. Le champ d'application englobe dorénavant non seulement les personnes pour qui le processus de la mort est amorcé, mais également les patients souffrant d'une maladie à l'issue probablement mortelle ainsi que des personnes qui souhaitent une aide médicale pour mettre fin à leur vie. Un chapitre concernant les principes éthiques explique le droit à l'autodétermination, le contexte social de l'autodétermination, les notions de «qualité de vie», de "souffrance» et de "soulagement de la souffrance». La clarification de l'objectif thérapeutique et la prise en charge des proches figurent également parmi les thèmes traités. Les chapitres suivants contiennent des indications concernant l'entretien sur la fin de vie et la mort et l'attitude à adopter face aux désirs de mourir. Le chapitre sur les processus 
de décision accorde une grande importance à la prise de décision partagée et à la planification anticipée du traitement et de la prise en charge.

Les «actes médicaux qui accélèrent - potentiellement ou certainement - la survenue de la mort» sont répartis en trois catégories: 1 . les actes généralement acceptés, tels que le renoncement et l'interruption des mesures de maintien en vie, le soulagement des douleurs et des autres symptômes ainsi que la sédation; 2 . les actes controversés comme l'assistance au suicide et le soutien au renoncement volontaire à l'alimentation et à l'hydratation; 3. les actes non autorisés, qui sont interdits par la loi. L'annexe des directives définit les objectifs thérapeutiques et les actes médicaux dans le contexte de la fin de vie et de la mort et contient des réflexions éthiques concernant les objectifs de la médecine.

\section{Actes controversés}

Les directives formulent des garde-fous éthiques pour les actes médicaux controversés. Contrairement aux mesures médicales généralement acceptées, il n'existe aucune indication médicale pour ces actes. Le médecin doit décider si l'assistance au suicide est conforme à son éthique professionnelle et si, dans le cas concret, il est convaincu d'agir pour le bien du patient. Une étude concernant l'attitude du corps médical face à l'assistance au suicide ${ }^{1}$, réalisée en 2014 à la demande de l'ASSM, a révélé que si de nombreux médecins considèrent l'assistance au suicide comme acceptable, seule une minorité est prête à la pratiquer elle-même. Les directives soulignent également qu'en aucun cas, le patient n'a le droit d'exiger une assistance au suicide et que chaque médecin est libre de décider s'il veut ou non envisager un tel acte.

Compte tenu des dispositions juridiques et des attitudes divergentes des médecins, des autres professionnels de la santé ainsi que de l'opinion publique face aux demandes de suicide, le dilemme entre les exigences éthiques d'encouragement de l'autodétermination et de protection de la vie ne peut être résolu. Il importe néanmoins de proposer aux professionnels de la santé des garde-fous aussi clairs que possible.

La formulation de critères médicaux objectifs pour l'admissibilité de l'assistance au suicide pose problème: d'une part, des questions délicates de délimitation (qu'est-ce qu'une maladie mortelle?) se poseraient et, d'autre part, les patients confrontés à une maladie mortelle ainsi que les soignants en charge de ces patients pourraient se sentir pressés de se justifier lorsqu'ils refusent d'envisager la possibilité d'une assistance au suicide. La plupart du temps, le désir auto- déterminé de suicide d'un patient n'est pas motivé par son état médical objectif, mais par la souffrance qu'il ressent subjectivement comme insupportable. Cette souffrance n'est toutefois pas objectivable. Pour justifier que l'assistance au suicide relève du domaine de compétence de la médecine, le patient doit présenter des symptômes de maladies identifiables ou des limitations fonctionnelles tangibles. Pour le médecin, la souffrance doit pouvoir être perçue de manière intersubjective.

\section{Réglementation de l'assistance au suicide}

Extrait des directives, chapitre 6.2.1:

"Aux termes de l'article 115 du code pénal, l'assistance au suicide n'est pas punissable lorsqu'elle intervient sans mobile égoïste. Cette règle s'applique à tout individu. Si un patient demande une assistance au suicide, il s'agit d'un désir de mourir qui doit être soigneusement évalué. Le patient doit être encouragé à parler de son désir de suicide avec ses proches.

Le rôle du médecin face à la fin de vie et à la mort consiste à soulager les symptômes et accompagner le patient. Il n'est de son devoir ni de proposer une assistance au suicide, ni de la pratiquer. L'assistance au suicide n'est pas un acte médical auquel les patients peuvent prétendre; il s'agit toutefois d'un acte admissible du point de vue juridique, pouvant être réalisé par le médecin s'il a la conviction que les conditions préalables énumérées ci-dessous sont remplies.

Font partie de l'assistance au suicide les actes accomplis dans l'intention de permettre à une personne capable de discernement de mettre fin à ses jours, notamment la prescription ou la délivrance de médicaments à des fins de suicide. La prescription d'un médicament destiné au suicide doit être signalée aux autorités cantonales compétentes dans un délai de 30 jours. Comme tout autre patient, celui qui souhaite mettre fin à ses jours avec l'aide d'une organisation d'assistance au suicide a un droit de regard dans son dossier et peut en demander une copie.

Si le patient le souhaite, le médecin traitant peut évaluer ses fonctions cognitives et, le cas échéant, établir une confirmation de sa capacité de discernement concernant les décisions de la vie quotidienne. Une telle évaluation ne correspond pas à une participation au suicide assisté. Le médecin peut également procéder à une évaluation et, le cas échéant, établir une confirmation de la capacité de discernement spécifiquement requise pour un suicide assisté (qui ne ressort pas de la capacité de discernement concernant les décisions de la vie quotidienne). Une telle évaluation ne peut toutefois pas être exigée par le patient. 
Si après une information et une évaluation minutieuses le patient persiste dans son désir, le médecin peut, sur la base d'une décision, dont il endosse personnellement la responsabilité, apporter une aide au suicide, sous réserve que cinq conditions préalables soient réunies et satisfaites. De plus, une tierce personne indépendante, qui ne doit pas nécessairement être médecin, doit examiner si les deux premières conditions listées ci-dessous sont remplies.

- La patiente est capable de discernement par rapport au suicide assisté. Le médecin doit documenter avec précision qu'il a exclu l'incapacité de discernement de la patiente. En cas de maladie psychique, de démence ou d'un autre état fréquemment associé à une incapacité de discernement, la capacité de discernement doit être évaluée par un spécialiste correspondant.

- Le désir de mourir est mûrement réfléchi, il ne résulte pas d'une pression extérieure et il est persistant. En cas de suspicion d'une relation de dépendance problématique, son influence possible sur le désir de suicide doit être examinée soigneusement.

- Les symptômes de la maladie et/ou les limitations fonctionnelles du patient lui causent une souffrance qu'il juge insupportable.

- Des options thérapeutiques indiquées ainsi que d'autres offres d'aide et de soutien ont été recherchées et ont échoué ou ont été jugées inacceptables par le patient capable de discernement à cet égard.

- Compte tenu de l'histoire du patient et après des entretiens répétés, le médecin considère que le souhait du patient de ne plus vouloir vivre cette situation de souffrances insupportables est compréhensible pour lui et peut, dans ce cas concret, estimer acceptable d'apporter une aide au suicide.
Le dernier geste du processus conduisant à la mort doit dans tous les cas être accompli par le patient lui-même. Un décès survenant suite à un suicide assisté doit être déclaré à l'autorité compétente comme décès pour cause non naturelle. Au préalable, pendant et avant le suicide assisté, il importe de prendre en compte les besoins des proches, mais également ceux de l'équipe de soins interprofessionnelle et de l'entourage, et de leur offrir le soutien nécessaire.»

\section{La préoccupation principale reste le bien du patient}

En cette époque d'évolution rapide et de vives controverses, les directives précisent les attentes envers les médecins et les autres professionnels de la santé dans le contexte de la fin de vie et de la mort. Elles visent à mettre en relation les différents points de vue et valeurs et veillent au respect et à la protection de l'autodétermination de toutes les parties prenantes patientes, proches et professionnels de la santé. Une attitude face à la fin de vie et à la mort qui se veut orientée selon le bien du patient doit veiller à ne pas solliciter excessivement les proches, ni à menacer l'éthique des professionnels de la santé.

Les directives sont disponibles en ligne en allemand, français, italien et anglais et peuvent être comma0ndées gratuitement sous forme de brochure (en allemand et en français): assm.ch/directives 